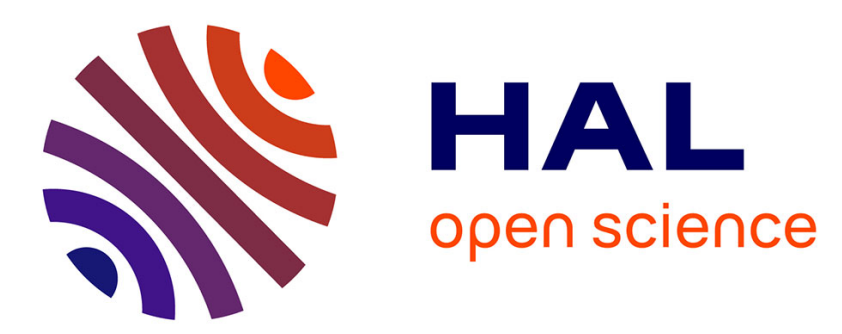

\title{
Electron pitch-angle diffusion in radiation belts: The effects of whistler wave oblique propagation
}

\author{
A Artemyev, O Agapitov, H Breuillard, V Krasnoselskikh, G Rolland
}

\section{To cite this version:}

A Artemyev, O Agapitov, H Breuillard, V Krasnoselskikh, G Rolland. Electron pitch-angle diffusion in radiation belts: The effects of whistler wave oblique propagation. Geophysical Research Letters, 2012, 39, L08105 (6 p.). 10.1029/2012GL051393 . insu-01179476

\section{HAL Id: insu-01179476 \\ https://hal-insu.archives-ouvertes.fr/insu-01179476}

Submitted on 22 Jul 2015

HAL is a multi-disciplinary open access archive for the deposit and dissemination of scientific research documents, whether they are published or not. The documents may come from teaching and research institutions in France or abroad, or from public or private research centers.
L'archive ouverte pluridisciplinaire HAL, est destinée au dépôt et à la diffusion de documents scientifiques de niveau recherche, publiés ou non, émanant des établissements d'enseignement et de recherche français ou étrangers, des laboratoires publics ou privés. 


\title{
Electron pitch-angle diffusion in radiation belts: The effects of whistler wave oblique propagation
}

\author{
A. Artemyev, ${ }^{1,2}$ O. Agapitov, ${ }^{1,3,4}$ H. Breuillard, ${ }^{1}$ V. Krasnoselskikh, ${ }^{1}$ and G. Rolland ${ }^{5}$
}

Received 16 February 2012; revised 21 March 2012; accepted 22 March 2012; published 21 April 2012.

[1] We calculated the electron pitch-angle diffusion coefficients in the outer radiation belt for $L$-shell $\sim 4.5$ taking into account the effects of oblique whistler wave propagation. The dependence of the distribution of the angle $\theta$ between the whistler wave vector and the background magnetic field on magnetic latitude is modeled after statistical results of Cluster wave angle observations. According to in-situ observations, the mean value and the variance of the $\theta$ distribution rapidly increase with magnetic latitude. We found that inclusion of oblique whistler wave propagation led to a significant increase in pitch-angle diffusion rates over those calculated under the assumption of parallel whistler wave propagation. The effect was pronounced for electrons with small equatorial pitch-angles close to the loss cone and could result in as much as an order of magnitude decrease of the electron lifetimes. We show that the intensification of pitch-angle diffusion can be explained by the contribution of higher order cyclotron resonances. By comparing the results of calculations obtained from two models of electron density distribution along field lines, we show that the effect of the intensification of pitch-angle diffusion is stronger when electron density does not vary along field lines. The intensification of pitch-angle diffusion and corresponding decrease of energetic electron lifetime result in significant modification of the rate of electron losses and should have an impact on formation and dynamics of the outer radiation belt. Citation: Artemyev, A., O. Agapitov, H. Breuillard, V. Krasnoselskikh, and G. Rolland (2012), Electron pitch-angle diffusion in radiation belts: The effects of whistler wave oblique propagation, Geophys. Res. Lett., 39, L08105, doi:10.1029/ 2012 GL051393.

\section{Introduction}

[2] Studies of particle energization and scattering in the inner magnetosphere began in the 1960's with pioneering work by Trakhtengerts [1966] and Kennel and Petschek [1966]. Pitch-angle and energy diffusion due to the resonant interaction of electrons with whistler waves are now considered to be one of the main mechanisms responsible for the dynamics of electron radiation belts (see, e.g., reviews by Shprits et al. [2008] and Thorne [2010]). The efficiency of particle acceleration due to wave-particle resonant interaction may be comparable or even more significant than effect of

\footnotetext{
${ }^{1}$ LPC2E/CNRS-University of Orleans, Orleans, France.

${ }^{2}$ Space Research Institute, RAS, Moscow, Russia.

${ }^{3}$ Astronomy and Space Physics Department, National Taras Shevchenko University of Kiev, Kiev, Ukraine.

${ }^{4}$ Le Studium, Institute for Advanced Studies, Orleans, France.

${ }^{5} \mathrm{CNES}$, Toulouse, France.
}

Copyright 2012 by the American Geophysical Union. 0094-8276/12/2012GL051393 the adiabatic energy increase (see the discussion of the relationship between these two mechanisms in the work of Horne et al. [2005a], Chen et al. [2007], and Shprits et al. [2009]).

[3] Since the work of Kennel and Petschek [1966], waveparticle resonant interaction within the inner magnetosphere has been described by a quasi-linear diffusion equation in pitch-angle and energy space. The description requires one to determine the coefficients of pitch-angle, $\alpha$, and the energy $E$ diffusion, $D_{\alpha \alpha}$ and $D_{E E}$, respectively. In order to calculate these coefficients one needs to define the wave spectrum characteristics. The widely used [see, e.g., Lyons et al., 1972; Glauert and Horne, 2005; Horne et al., 2005b; Ni et al., 2011, and references therein] approach consists of an introduction of the wave frequency distribution $\hat{B}^{2}(\omega)$ and the distribution $g(X)$ for the variable $X=\tan \theta$ that has a single narrow peak, where $\theta$ is the angle between the wave vector direction and the background magnetic field. The calculations are based on the assumption that diffusion processes are slow with respect to the bounce oscillations of trapped particles between mirror points. For this case, one needs to average out the diffusion coefficients over all latitudes for a given $L$-shell.

[4] To evaluate the diffusion coefficients, the distributions $\hat{B}^{2}(\omega)$ and $g(X)$ need to be defined either self-consistently from theoretical models or determined from experimental observations. For this work, we use the second approach. Both distributions are often assumed to be Gaussian with the following two parameters: the mean value and the variance.

[5] For the frequency distribution $\hat{B}^{2}(\omega)$, the parameters are generally defined as input data [Glauert and Horne, 2005; Horne et al., 2005b; Summers et al., 2007b] or can be determined from observations [see, e.g., Ni et al., 2011]. Although Shprits and Ni [2009] and Ni et al. [2011] demonstrated the role of the obliqueness of wave propagation for pitch-angle diffusion, thus far the overwhelming majority of calculations for diffusion coefficients have been made by assuming the parallel propagation of whistler waves, when the mean value of $X$ is equal to zero [Glauert and Horne, 2005; Horne et al., 2005b; Albert, 2007]. Early spacecraft observations showed that angle $\theta$ is small enough in the vicinity of the equator [Hayakawa et al., 1984; Goldstein and Tsurutani, 1984]. Summers [2005], utilizing an assumption for wave parallel propagation, developed a simplified technique for diffusion coefficient calculations and later generalized the technique by including the effects of heavy ions in the dispersion relationship [Summers et al., 2007b]. The approximation of the wave parallel propagation is widely applied in order to obtain analytical estimates of diffusion coefficients [see Albert, 2007; Mourenas and Ripoll, 2012, and references therein]. 


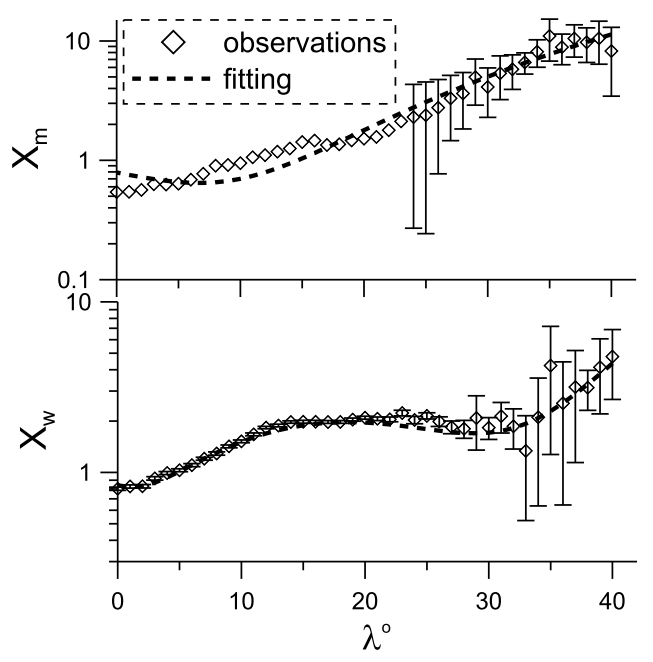

Figure 1. The dependencies of the mean value $X_{m}$, and the variance $X_{w}$ on magnetic latitude $\lambda$ are shown by diamonds (experimental data). The corresponding fitting used for modeling is shown using dotted curves.

[6] Significant deviations in $\theta$ from zero for particular events were detected by Lauben et al. [2002] and Santolik et al. [2009]. Additionally, recent spacecraft statistical observations [Haque et al., 2010; Agapitov et al., 2011; Li et al., 2011] and numerical modeling [Bortnik et al., 2011] have unambiguously demonstrated that the direction of the whistler wave-vector can substantially deflect from the local direction of the magnetic field, even at relatively low latitudes. The deflection becomes significant for magnetic latitudes equal or larger than $\lambda>15^{\circ}$, where whistler waves can have wave vectors close to the resonant cone, approaching the perpendicular of the background magnetic field [Agapitov et al., 2011].

[7] In this work, we modified the method for diffusion coefficient calculations as proposed by Glauert and Horne [2005] by incorporating a realistic dependence of the $g(X)$ distribution on the magnetic latitude $\lambda$. We used the results of the statistical study of whistler wave propagation [Agapitov et al., 2011] to define the distribution $g_{\lambda}(X)$ as a function of $\lambda$ for the computation of bounce averaged coefficients in pitch-angle diffusion $\left\langle D_{\alpha \alpha}\right\rangle$. Here, we show that a consideration of the realistic distribution of $g_{\lambda}(X)$ results in an increase of the role of a higher number of cyclotron resonances, and leads to the important growth of $\left\langle D_{\alpha \alpha}\right\rangle$.

\section{The Statistical Distribution of Whistler Propagation Directions}

[8] For calculating diffusion rates, we used a Gaussian distribution of the variable $X=\tan \theta$, similar to previous studies [see, e.g., Glauert and Horne, 2005; Horne et al., 2005b; Ni et al., 2011, and references therein]: $g(X)=$ $\exp \left(-\left(X-X_{m}\right)^{2} / X_{w}^{2}\right)$. However, in contrast to these models we considered the mean value $X_{m}$, and the variance $X_{w}$ to be functions of the magnetic latitude $\lambda$. According to Cluster statistics observations, the mean value, and the variance of the $\theta$ distribution increase with magnetic latitude [see Agapitov et al., 2011, Figure 2(e)]. On the equatorial plane, Agapitov et al. [2011] found $X_{m} \approx 0.3$ and $X_{w} \approx 0.8$, with $X_{m} \approx 4$ and $X_{w} \approx 2$ already at $\lambda=30^{\circ}$.
We transformed the observational distribution of $\theta$ from Agapitov et al. [2011] to the distribution of the $X$ variable and approximated this new distribution using a Gaussian function. The dependence of the parameters of this approximation (the mean value and the variance $X_{m}, X_{w}$ ) on $\lambda$ were fitted using the following polynomial functions:

$$
\begin{aligned}
& X_{m}=0.79-0.36 l+0.11 l^{2}+0.16 l^{3} \\
& X_{w}=\sqrt{2}\left(0.84-0.4 l+2.0 l^{2}-1.1 l^{3}+0.17 l^{4}\right)
\end{aligned}
$$

where $l=\lambda / 10^{\circ}$ and $\lambda<40^{\circ}$. A comparison of equation (1) with the dependencies obtained directly from Cluster observations is presented in Figure 1. Here, the experimental data were collected for the $L$-shells $\in[3.5,5.5]$.

\section{Pitch-Angle Diffusion Coefficients}

[9] We calculated the local diffusion coefficients using the following expression obtained by Glauert and Horne [2005]:

$$
D_{\alpha \alpha}=\sum_{i, n} \int_{X_{\min }}^{X_{\max }}\left[\frac{n \Omega_{e} / \gamma+\omega_{i, n} \sin ^{2} \alpha}{\cos \alpha}\right]^{2} \frac{g(X) \hat{B}^{2}\left(\omega_{i, n}\right)}{N\left(\omega_{i, n}\right)} \Delta_{i, n} d X
$$

where $\Delta_{i, n}$ is the resonant factor $\sim\left|v_{\|}-\partial \omega / \partial k_{\|}\right|^{-1}$ (see details in work by Glauert and Horne [2005]), and $\omega_{i, n}$ are the resonance frequencies, and, as follows:

$$
\begin{aligned}
& N(\omega)=\left.\frac{1}{2 \pi^{2}} \int_{X_{\min }}^{X_{\max }} \frac{g(X) k_{i, n}^{2} X}{\left(1+X^{2}\right)^{3 / 2}} \frac{\partial k}{\partial \omega}\right|_{X} d X, \\
& \hat{B}^{2}(\omega)=A \exp \left(-\left(\frac{\omega-\omega_{m}}{\delta w}\right)^{2}\right), \quad \omega \in\left[\omega_{l c}, \omega_{u c}\right],
\end{aligned}
$$

where $\hat{B}^{2}=0$ for $\omega \notin\left[\omega_{l c}, \omega_{u c}\right], \omega_{l c, u c}=\omega_{m} \mp 1.5 \delta \omega$, and $A$ is determined from the normalization $\int \hat{B}^{2}(\omega) d \omega=B_{w}^{2}$. The boundary values of $X$ are $X_{\min }=0$ and $X_{\max }=\min \left(10, X_{r}\right)$, where $X_{r}$ is the $X$ value in the resonant cone angle (see, e.g., the discussion in work by Glauert and Horne [2005]). The resonant frequencies (and the corresponding wavenumbers $k_{i, n}$ ) can be found from the dispersion relationship $\omega=\omega(k)$ and the resonant condition $\omega-k_{\|} v_{\|}=-n \Omega_{e} / \gamma$; where $\Omega_{e}=|e| B / m_{e} c, v_{\|}=c \sqrt{1-\gamma^{-2}} \cos \alpha, \gamma$ is the gamma factor, and $i$ is the number of resonant roots. Although, recent calculations demonstrate possibly important roles for the difference between the real background magnetic field and the dipole model [Orlova and Shprits, 2010; Ni et al., 2011], we used a dipole approximation to obtain first estimates and to compare them with previous calculations, as follows: $B=B_{e q} b(\lambda), \mathrm{b}(\lambda)=\sqrt{1+3 \sin ^{2} \lambda} / \cos ^{6} \lambda$, and the equatorial value $B_{e q}$ corresponds to the selected $L$-shell. Here, $\Omega_{e q}=|e| B_{e q} / m_{e c}$ indicates the equatorial gyrofrequency.

[10] We solved the resonant equations for each latitude $\lambda$ and found $\omega_{i, n}(\lambda, \alpha, \gamma, s)$. The ratio of the plasma frequency and the gyrofrequency $s=\omega_{p e} / \Omega_{e}$ should be specified by choosing $\omega_{p e}$ as a function of $\lambda$. For the majority of calculations, a constant approximation $\omega_{p e}=$ const was used [Glauert and Horne, 2005; Ni et al., 2011; Summers et al., 2007b]. However, a possible increase in the electron density with latitude [Denton et al., 2002] resulted in an increase in $\omega_{p e}$. Here, we used the following two approximations: 1) $\omega_{p e}=$ const for the given $L$-shell, and 2) the ratio $s$ that 

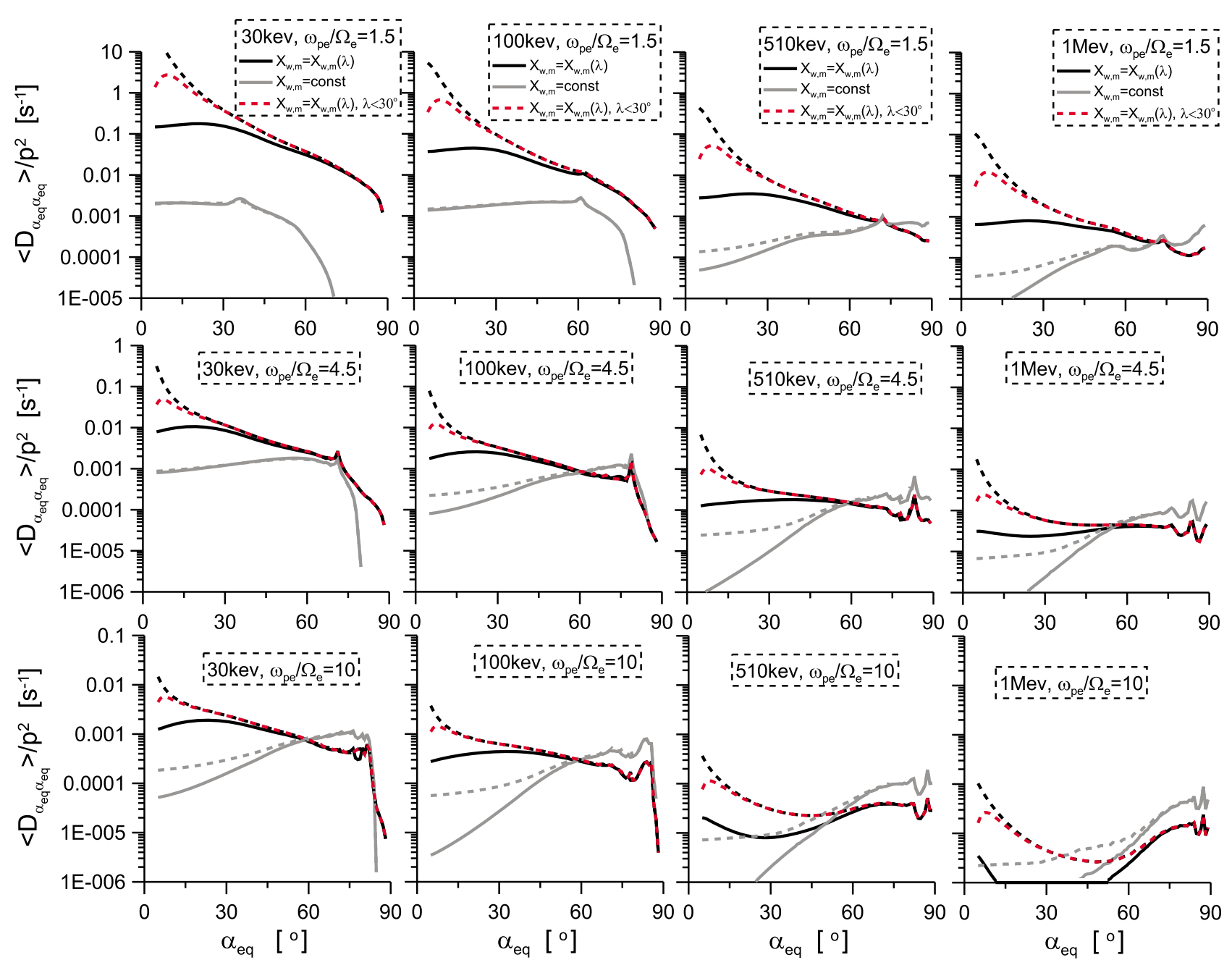

Figure 2. Pitch-angle diffusion coefficients averaged over electron bounce oscillations are shown for constant parameters $\left(X_{w}=0.577, X_{m}=0\right.$, gray color) and for parameters dependent on the magnetic latitude $\left(X_{m, w}=X_{m, w}(\lambda)\right.$ from equation (1), black color). Dotted curves correspond to the approximation $\omega_{p e}=$ const, and solid curves show results for $s=$ const. The corresponding particle energy and the ratio $\omega_{p e} / \Omega_{e}$ evaluated at the equator are indicated inside the panels. Red dotted curve shows diffusion coefficient calculated with $X_{m, w}=X_{m, w}(\lambda)$ and $\omega_{p e}=$ const for $\lambda_{m}=\min \left(\lambda_{\max }, 30^{\circ}\right)$.

is assumed to be constant for a given $L$-shell (i.e. $\omega_{p e}$ varies in a similar manner with $\Omega_{e}$ ). Real variation in the $s$ parameter should be determined between these two approximations.

[11] Then, for each $\lambda$ we calculated the local value of the diffusion coefficient $D_{\alpha \alpha}(\alpha, \lambda)$ using $X_{m}(\lambda)$ and $X_{w}(\lambda)$ from equation (1). The coefficient $\left\langle D_{\alpha_{e q} \alpha_{e q}}\right\rangle$ was obtained by averaging over magnetic latitudes (corresponding to the averaging obtained over bounce oscillations), as follows:

$$
\left\langle D_{\alpha_{e q} \alpha_{e q}}\right\rangle=\frac{1}{T} \int_{0}^{\lambda_{m}} D_{\alpha \alpha}(\alpha, \lambda) \frac{\cos \alpha \cos ^{7} \lambda}{\cos ^{2} \alpha_{e q}} d \lambda,
$$

where $\sin ^{2} \alpha=b(\lambda) \sin ^{2} \alpha_{e q}, T=1.30-0.56 \sin \alpha_{e q}, \lambda_{m}=$ $\min \left(\lambda_{\max }, 40^{\circ}\right), b\left(\lambda_{\max }\right) \sin ^{2} \alpha_{e q}=1$, and $\alpha_{e q}$ is the equatorial value of the pitch-angle (see details in work by Glauert and Horne [2005]).

[12] We also calculated the diffusion coefficients $\left\langle D_{\alpha_{e q} \alpha_{e q}}\right\rangle$ using $X_{w}=0.577$ and $X_{m}=0$ for a comparison with results found by Glauert and Horne [2005] and Summers et al. [2007b]. For calculating these coefficients, we used $\lambda_{m}=$ $\lambda_{\max }, X_{\min }=0, X_{\max }=1$.
[13] For both calculations we used the wave magnetic field amplitude $B_{w}=100 \mathrm{pT}$ and $\omega_{m}=0.35 \Omega_{e q}$ similar to the previous studies. The $L$-shell was 4.5 and maximum number of cyclotron harmonics was taken to be equal to $n= \pm 5$ (we used the same parameters used by Glauert and Horne [2005]). The pitch-angle diffusion coefficients were normalized on $p^{2}=\left(\gamma^{2}-1\right) m_{e c}^{2}$.

[14] In Figure 2, we present the results of the calculations for the diffusion coefficients with constant $X_{w, m}$ and with $X_{w, m}=X_{w, m}(\lambda)$ as described by equation (1). The main differences between the coefficients were found for particles with a small pitch-angle at the equator $\alpha_{e q}$. Indeed, these particles can reach high latitudes $\left(\lambda_{\max }>15^{\circ}\right)$, where values of $X_{m}$ and $X_{w}$ are substantially larger than for the equatorial plane $\lambda=0^{\circ}$. The ratio of $\left\langle D_{\alpha_{e q} \alpha_{e q}}\right\rangle$ for $X_{w, m}=$ const and for $X_{w, m}=X_{w, m}(\lambda)$ from equation (1) can exceed an order of magnitude. In comparison with Shprits and $N i$ [2009] and Ni et al. [2011], where a nonzero $X_{m}$ was also used, we obtained a more substantial increase of $\left\langle D_{\alpha_{e q} \alpha_{e q}}\right\rangle$ due to the increase of variance $X_{w}$ with latitude. Comparison of diffusion coefficients calculated with $\lambda_{m}=\min \left(\lambda_{\max }, 30^{\circ}\right)$ and with $\lambda_{m}=$ $\min \left(\lambda_{\max }, 40^{\circ}\right)$ show the role of resonant wave-particle 

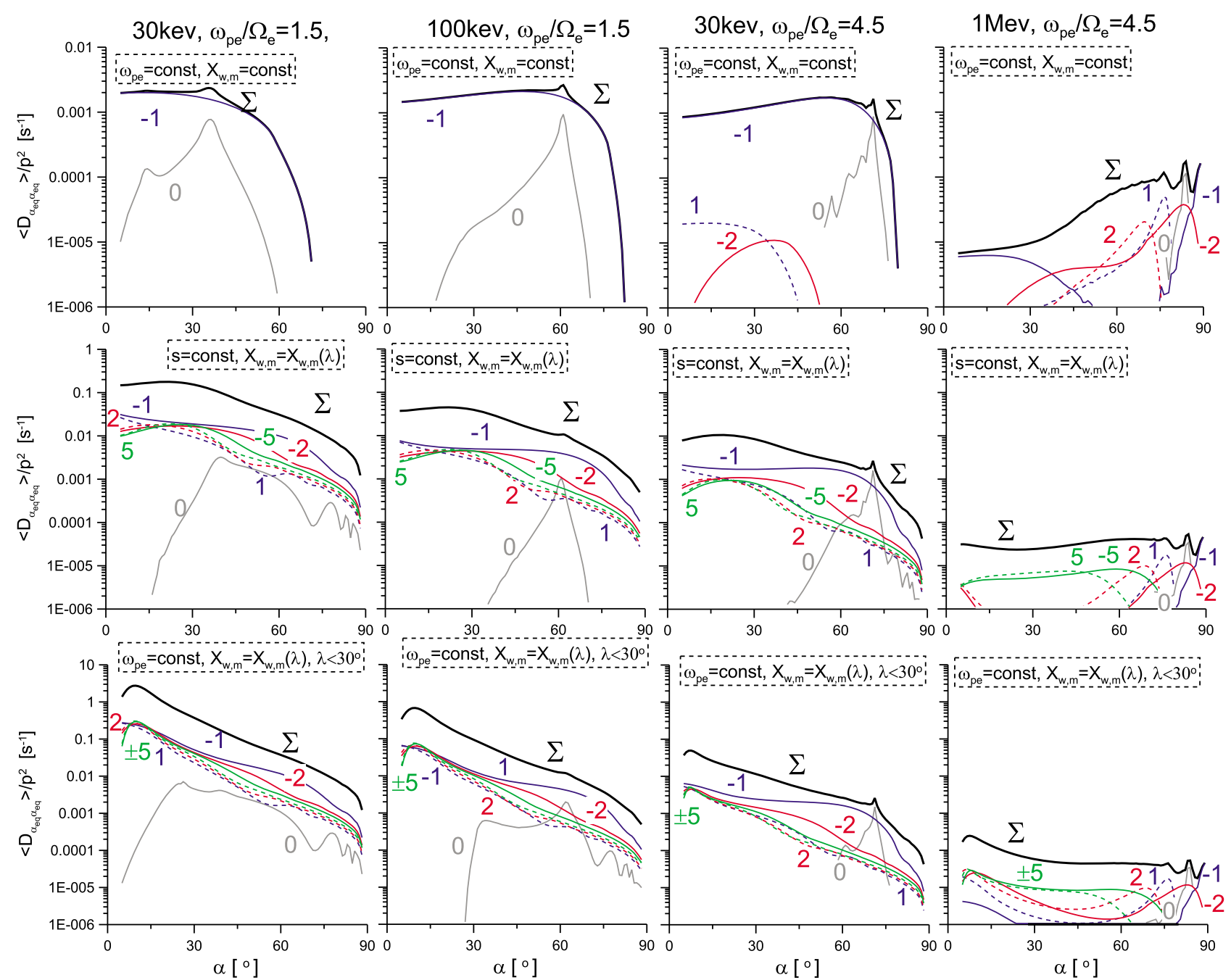

Figure 3. The pitch-angle diffusion coefficients, averaged over electron bounce oscillations for each cyclotron resonance $n=0, \pm 1, \pm 2, \pm 5$, are shown using colored curves. The total value of the diffusion coefficient $\left\langle D_{\alpha_{e q} \alpha_{e q}}\right\rangle=\sum_{n}\left\langle D_{\alpha_{e q}}^{n} \alpha_{e q}\right\rangle$ is marked by the symbol $\Sigma$.

interaction for small pitch-angles. The strong increase of pitchangle diffusion coefficients for $\alpha_{e q}<10^{\circ}$ corresponds with resonant interaction with almost transverse waves at $\lambda>30^{\circ}$.

\section{Higher Order Cyclotron Resonances}

[15] To determine the importance of the contribution of higher order cyclotron resonances to $\left\langle D_{\alpha_{e q} \alpha_{e q}}\right\rangle$ we plotted the total diffusion coefficients $\left\langle D_{\alpha_{e q} \alpha_{e q}}\right\rangle=\sum_{n}\left\langle D_{\alpha_{e q}}^{n} \alpha_{e q}\right\rangle$ and the partial rates $\left\langle D_{\alpha_{e q} \alpha_{e q}}^{n}\right\rangle$ for various conditions in Figure 3.

[16] One can see that for $X_{m, w}=$ const, only resonances with $n=0$ (Cherenkov resonance) and $n=-1$ play an essential role. In contrast, higher order resonances significantly contribute to pitch-angle diffusion coefficients in the system, with $X_{w, m}=X_{w, m}(\lambda)$. The effect of the increase in the role played by higher order resonances for oblique whistler waves was noted before by Shklyar and Matsumoto [2009]. One can determine that different higher order resonances tend to be dominant at various equatorial pitch angle intervals, showing an energy dependence (a similar effect was observed for calculations with a nonzero $X_{m}$ by Shprits and $\mathrm{Ni}$ [2009]).
[17] To point out the intensification of the role of higher order resonances, let us suppose that the distribution dependence of $\hat{B}^{2}(\omega)$ on the frequency is sufficiently narrow, with $\omega_{i, n} \approx \omega_{m}$ (see the details of this approach in work by Mourenas and Ripoll [2012]). Then, the resonance conditions, together with the simplified dispersion relationship, allow one to evaluate the largest possible value of the resonance order for which wave particle interaction occurs.

$$
n^{2} \approx \frac{\omega_{p e}^{2}}{\Omega_{e}^{2}} \frac{\omega_{m}\left(v_{\|} \gamma / c\right)^{2} \cos ^{2} \theta}{\Omega_{e} \cos \theta-\omega_{m}}
$$

[18] One can see that higher order resonances become important when the corresponding vector approaches the resonance cone for the given frequency $\cos \theta=\omega_{m} / \Omega_{e}$ at a given latitude. On the other hand, for the quasi-parallel propagation, the angle $\theta$ for any given $\lambda$ remains far from the resonance cone. One can conclude that for this case, higher order resonances cannot provide any significant input to the diffusion coefficients (at least for small particle energy, when $\gamma \sim 1)$. 
[19] According to experimental observations, $X_{m}$ and $X_{w}$ grow with $\lambda$ (see Figure 1 and Agapitov et al. [2011]). For such a case, $\cos \theta(\lambda)$, corresponding to the core of the distribution $g_{\lambda}(X)$, decreases with $\lambda$ while the wave vector becomes closest to the resonant cone. As a result, higher order resonances can contribute to diffusion. To reach latitudes with a small $\cos \theta$, particles should have small equatorial pitch angles, in agreement with the results shown in Figures 2 and 3.

\section{Discussion and Conclusions}

[20] In this work we show that higher order cyclotron resonances can contribute significantly to electron pitchangle diffusion (for small $\alpha_{e q}$ ) where whistler wave-vector directions become oblique to the background magnetic field. Although our results demonstrate a significant increase in the pitch-angle diffusion coefficients for small equatorial pitchangles $\alpha_{e q}$, one would not expect a comparable increase in the energy diffusion rate. For energy diffusion coefficients, one can find $\left\langle D_{E E}^{n}\right\rangle \sim\left\langle D_{\alpha_{e q} \alpha_{e q}}^{n} / n^{2}\right\rangle$ [see, e.g., Glauert and Horne, 2005; Summers et al., 2007b]. As a result, the impact of higher order resonances is weaker for energy diffusion, and the expected increase in energy diffusion rates should be smaller than the increase obtained for pitch-angle diffusion rates.

[21] For our calculations, we employed the distribution of the variable $X=\tan \theta$, as first proposed by Lyons et al. [1972]. The issue is important to note since the transformation of the $\theta$ to $X$ variable is nonlinear and the real dependence of $g_{\lambda}(X)$ on $X$ significantly deviates from Gaussian. Therefore, one can consider two possible approaches for describing $g_{\lambda}(X)$, as seen in spacecraft observations with slightly different results. On one hand, we can find the mean value $\langle\theta\rangle$ and the variance $\Delta \theta$ of the $\theta$ distribution, and then assume $X_{m}=$ $\tan \langle\theta\rangle$ and $X_{w}=\tan \Delta \theta$ [see, e.g., Glauert and Horne, 2005; Ni et al., 2011]. On the other hand, we can transform the observed $\theta$ distribution onto the distribution of the $X$ variable and then calculate $X_{m, w}$ as a parameter of the approximation of this new distribution using the Gaussian function. The choice of these two approaches is not important for constant small values of $\langle\theta\rangle$ and $\Delta \theta$. For $\langle\theta\rangle$, depending on $\lambda$, it seems to be more relevant to rewrite $g_{\lambda}(X)$ as a distribution of the angle $\theta$ and to use the Gaussian (or a more complicated) approximation for $g_{\lambda}(\theta)$. Such an improvement is beyond the scope of this work and is left for future publications.

[22] Pitch-angle diffusion rates determine the lifetime of electrons in radiation belts and define the evolution of particle flux [see Horne et al., 2005b; Summers et al., 2007a; Shprits et al., 2009, and references therein]. According to simplified estimates by Albert and Shprits [2009], the electron lifetime is $\sim 1 /\left\langle D_{\alpha_{e q} \alpha_{e q}}\right\rangle$, where diffusion rate is evaluated at the loss-cone boundary pitch-angle. Therefore, the increase of $\left\langle D_{\alpha_{e q} \alpha_{e q}}\right\rangle$ obtained for particles with small equatorial pitchangles (close to loss-cone) should result in a significant decrease in lifetime.

[23] In conclusion, we calculated pitch-angle diffusion coefficients for the realistic distribution of $X=\tan \theta$ while taking into account the dependence of this distribution on magnetic latitude. The obtained results demonstrate that the diffusion rates of electrons with small pitch-angles are significantly larger for the realistic $g_{\lambda}(X)$ distribution, in comparison with the approximation of parallel wave propagation, when $X_{m}=0$. In this work, we demonstrated that the increase in diffusion rates corresponds to an important contribution of higher order cyclotron resonances.

[24] Acknowledgments. Authors are thankful to D. Mourenas for fruitful discussions and to both reviewers for useful comments and suggestions. This work was supported by CNES through the grant "Modele d'ondes". Work of A.O. was supported by the STUDIUM program of the Region Centre in France. Authors thank the ESA Cluster Active Archive for providing the STAFF-SA data set.

[25] The Editor thanks two anonymous reviewers for their assistance in evaluating this paper.

\section{References}

Agapitov, O., V. Krasnoselskikh, Y. V. Khotyaintsev, and G. Rolland (2011), A statistical study of the propagation characteristics of whistler waves observed by Cluster, Geophys. Res. Lett., 38, L20103, doi:10.1029/2011GL049597.

Albert, J. M. (2007), Simple approximations of quasi-linear diffusion coefficients, J. Geophys. Res., 112, A12202, doi:10.1029/2007JA012551.

Albert, J. M., and Y. Y. Shprits (2009), Estimates of lifetimes against pitch angle diffusion, J. Atmos. Sol. Terr. Phys., 71, 1647-1652, doi:10.1016/j. jastp.2008.07.004.

Bortnik, J., L. Chen, W. Li, R. M. Thorne, and R. B. Horne (2011), Modeling the evolution of chorus waves into plasmaspheric hiss, J. Geophys. Res., 116, A08221, doi:10.1029/2011JA016499.

Chen, Y., G. D. Reeves, and R. H. W. Friedel (2007), The energization of relativistic electrons in the outer Van Allen radiation belt, Nat. Phys., 3, 614-617, doi:10.1038/nphys655.

Denton, R. E., J. Goldstein, and J. D. Menietti (2002), Field line dependence of magnetospheric electron density, Geophys. Res. Lett., 29(24), 2205, doi:10.1029/2002GL015963.

Glauert, S. A., and R. B. Horne (2005), Calculation of pitch angle and energy diffusion coefficients with the PADIE code, J. Geophys. Res., 110, A04206, doi:10.1029/2004JA010851.

Goldstein, B. E., and B. T. Tsurutani (1984), Wave normal directions of chorus near the equatorial source region, J. Geophys. Res., 89, 2789-2810, doi:10.1029/JA089iA05p02789.

Haque, N., M. Spasojevic, O. Santolík, and U. S. Inan (2010), Wave normal angles of magnetospheric chorus emissions observed on the Polar spacecraft, J. Geophys. Res., 115, A00F07, doi:10.1029/2009JA014717.

Hayakawa, M., Y. Yamanaka, M. Parrot, and F. Lefeuvre (1984), The wave normals of magnetospheric chorus emissions observed on board GEOS 2, J. Geophys. Res., 89, 2811-2821, doi:10.1029/JA089iA05p02811.

Horne, R. B., et al. (2005a), Wave acceleration of electrons in the Van Allen radiation belts, Nature, 437, 227-230, doi:10.1038/nature03939.

Horne, R. B., R. M. Thorne, S. A. Glauert, J. M. Albert, N. P. Meredith, and R. R. Anderson (2005b), Timescale for radiation belt electron acceleration by whistler mode chorus waves, J. Geophys. Res., 110, A03225, doi:10.1029/2004JA010811.

Kennel, C. F., and H. E. Petschek (1966), Limit on stably trapped particle fluxes, J. Geophys. Res., 71, 1.

Lauben, D. S., U. S. Inan, T. F. Bell, and D. A. Gurnett (2002), Source characteristics of ELF/VLF chorus, J. Geophys. Res., 107(A12), 1429, doi:10.1029/2000JA003019.

Li, W., J. Bortnik, R. M. Thorne, and V. Angelopoulos (2011), Global distribution of wave amplitudes and wave normal angles of chorus waves using THEMIS wave observations, J. Geophys. Res., 116, A12205, doi:10.1029/2011JA017035.

Lyons, L. R., R. M. Thorne, and C. F. Kennel (1972), Pitch-angle diffusion of radiation belt electrons within the plasmasphere, J. Geophys. Res., 77, 3455-3474, doi:10.1029/JA077i019p03455.

Mourenas, D., and J.-F. Ripoll (2012), Analytical estimates of quasi-linear diffusion coefficients and electron lifetimes in the inner radiation belt, J. Geophys. Res., 117, A01204, doi:10.1029/2011JA016985.

Ni, B., R. M. Thorne, N. P. Meredith, Y. Y. Shprits, and R. B. Horne (2011), Diffuse auroral scattering by whistler mode chorus waves: Dependence on wave normal angle distribution, J. Geophys. Res., 116, A10207, doi:10.1029/2011JA016517.

Orlova, K. G., and Y. Y. Shprits (2010), Dependence of pitch-angle scattering rates and loss timescales on the magnetic field model, Geophys. Res. Lett., 37, L05105, doi:10.1029/2009GL041639.

Santolík, O., D. A. Gurnett, J. S. Pickett, J. Chum, and N. CornilleauWehrlin (2009), Oblique propagation of whistler mode waves in the chorus source region, J. Geophys. Res., 114, A00F03, doi:10.1029/ 2009JA014586.

Shklyar, D., and H. Matsumoto (2009), Oblique whistler-mode waves in the inhomogeneous magnetospheric plasma: Resonant interactions with 
energetic charged particles, Surv. Geophys., 30, 55-104, doi:10.1007/ s10712-009-9061-7.

Shprits, Y. Y., and B. Ni (2009), Dependence of the quasi-linear scattering rates on the wave normal distribution of chorus waves, J. Geophys. Res., 114, A11205, doi:10.1029/2009JA014223.

Shprits, Y. Y., D. A. Subbotin, N. P. Meredith, and S. R. Elkington (2008), Review of modeling of losses and sources of relativistic electrons in the outer radiation belt II: Local acceleration and loss, J. Atmos. Sol. Terr. Phys., 70, 1694-1713, doi:10.1016/j.jastp.2008.06.014.

Shprits, Y. Y., D. Subbotin, and B. Ni (2009), Evolution of electron fluxes in the outer radiation belt computed with the VERB code, J. Geophys. Res., 114, A11209, doi:10.1029/2008JA013784.

Summers, D. (2005), Quasi-linear diffusion coefficients for field-aligned electromagnetic waves with applications to the magnetosphere, J. Geophys. Res., 110, A08213, doi:10.1029/2005JA011159.

Summers, D., B. Ni, and N. P. Meredith (2007a), Timescales for radiation belt electron acceleration and loss due to resonant wave-particle interactions: 2. Evaluation for VLF chorus, ELF hiss, and electromagnetic ion cyclotron waves, J. Geophys. Res., 112, A04207, doi:10.1029/ 2006JA011993.

Summers, D., B. Ni, and N. P. Meredith (2007b), Timescales for radiation belt electron acceleration and loss due to resonant wave-particle interactions: 1. Theory, J. Geophys. Res., 112, A04206, doi:10.1029/ 2006JA011801.

Thorne, R. M. (2010), Radiation belt dynamics: The importance of waveparticle interactions, Geophys. Res. Lett., 37, L22107, doi:10.1029/ 2010GL044990.

Trakhtengerts, V. Y. (1966), Stationary states of the Earth's outer radiation zone, Geomagn. Aeron., 6, 827-836.

O. Agapitov, H. Breuillard, and V. Krasnoselskikh, LPC2E/CNRSUniversity of Orleans, Orleans F-45071, France.

A. Artemyev, Space Research Institute, RAS, Moscow 117997, Russia. (ante0226@yandex.ru)

G. Rolland, CNES, Toulouse F-31401, France. 